\title{
Long-term dietary patterns and carotid artery intima media thickness: The Cardiovascular Risk in Young Finns Study
}

\author{
Vera Mikkilä $^{1 *}$, Leena Räsänen ${ }^{1}$, Marika M. L. Laaksonen ${ }^{1}$, Markus Juonala ${ }^{2}$, Jorma Viikari ${ }^{3}$, \\ Pirjo Pietinen ${ }^{4}$ and Olli T. Raitakari ${ }^{5}$ \\ ${ }^{1}$ Division of Nutrition, University of Helsinki, Helsinki, Finland \\ ${ }^{2}$ Research Centre of Applied and Preventive Cardiovascular Medicine, University of Turku, Turku, Finland \\ ${ }^{3}$ Department of Medicine, University of Turku, Turku, Finland \\ ${ }^{4}$ Department of Health Promotion and Chronic Disease Prevention, National Institute for Health and Welfare, Helsinki, Finland \\ ${ }^{5}$ Department of Clinical Physiology, University of Turku, Turku, Finland
}

(Received 22 September 2008 - Revised 17 February 2009 - Accepted 25 April 2009 - First published online 8 October 2009)

\begin{abstract}
A whole-diet approach has proven useful for characterising dietary exposure in cardiovascular epidemiology research. In our previous analyses, we found dietary patterns to be significant determinants of CVD risk factor levels among the Cardiovascular Risk in Young Finns cohort. We investigated the associations of major dietary patterns with carotid intima media thickness (IMT), a subclinical predictor of CVD, in healthy adults. The Young Finns Study is an ongoing, prospective cohort study with a 21-year follow-up to date. The subjects were children and adolescents at baseline in 1980 (aged 3-18 years), and all had reached adulthood by the latest follow-up in 2001 (aged 24-39 years). Complete dietary data from the years 1980, 1986 and 2001 and outcome data from the year 2001 were obtained from 785 subjects. The long-term average pattern score for a traditional dietary pattern (characterised by high consumption of rye, potatoes, butter, sausages, milk and coffee) was associated with IMT especially among subjects with a low score for the health-conscious dietary pattern (characterised by high consumption of vegetables, legumes and nuts, rye, tea, cheese and other dairy products). In multivariable regression analyses using long-term pattern scores as predictors, the traditional dietary pattern was independently associated with IMT in men $(P<0 \cdot 01)$, but not in women $(P=0 \cdot 66)$. Long-term adherence to traditional food choices seems to increase the risk of developing subclinical atherosclerosis among Finnish men.
\end{abstract}

CVD risk factors: Carotid artery intima media thickness: Dietary patterns: Cardiovascular Risk in Young Finns Study

The Cardiovascular Risk in Young Finns is a longitudinal study on CVD risk factors and their determinants among children and young adults in Finland ${ }^{(1)}$. The first crosssectional study was carried out in 1980, when the subjects were aged 3-18 years. The same subjects have been followed since, and by the follow-up in 2001, all subjects had reached adulthood. The study protocol has included extensive dietary assessments from the beginning.

Nutritional epidemiology is increasingly shifting its focus from a reductionist single-nutrient concept to a more comprehensive whole-diet approach, exploratory dietary pattern analyses being the most utilised method ${ }^{(2)}$. Particularly in research of dietary determinants of multifactorial noncommunicable diseases, such as cardiovascular diseases, both observational and experimental settings have shown promising results of the utility of the whole-diet approach ${ }^{(3-7)}$.

Carotid artery intima media thickness (IMT) is a surrogate marker of subclinical atherosclerosis that has been shown to predict cardiovascular mortality and morbidity ${ }^{(8)}$. Adulthood measures of carotid IMT correlate with several established cardiovascular risk factors among the Young Finns cohort $^{(9,10)}$. Diet has been shown to be one of the predictors of IMT (for example, Millen et al. ${ }^{(5)}$; Nettleton et al. $\left.{ }^{(7)}\right)$. In our previous analysis among the Young Finns cohort, we identified two major dietary patterns in the cohort, namely traditional and health-conscious patterns ${ }^{(11)}$. Very similar dietary patterns were identified at the three study points (baseline in 1980, 6-year follow-up in 1986, and 21-year follow-up in 2001; patterns in 2001 shown in Fig. 1). We observed substantial tracking of these patterns reflecting food choices from childhood to adulthood ${ }^{(11)}$. In further longitudinal analyses, traditional dietary pattern score was found to be positively associated with several CVD risk factors, while those with high health-conscious pattern scores had lower risk factor levels ${ }^{(12)}$.

The aim of the present study was to examine the associations of the previously identified dietary patterns with carotid IMT in this Finnish population cohort using repeated dietary measurements from childhood to adulthood. To our knowledge, this is the first study on the relationships between diet and IMT with a long-term whole-diet approach from childhood on.

Abbreviation: IMT, intima media thickness.

* Corresponding author: Dr Vera Mikkilä, fax +3589191 58269, email vera.mikkila@helsinki.fi 


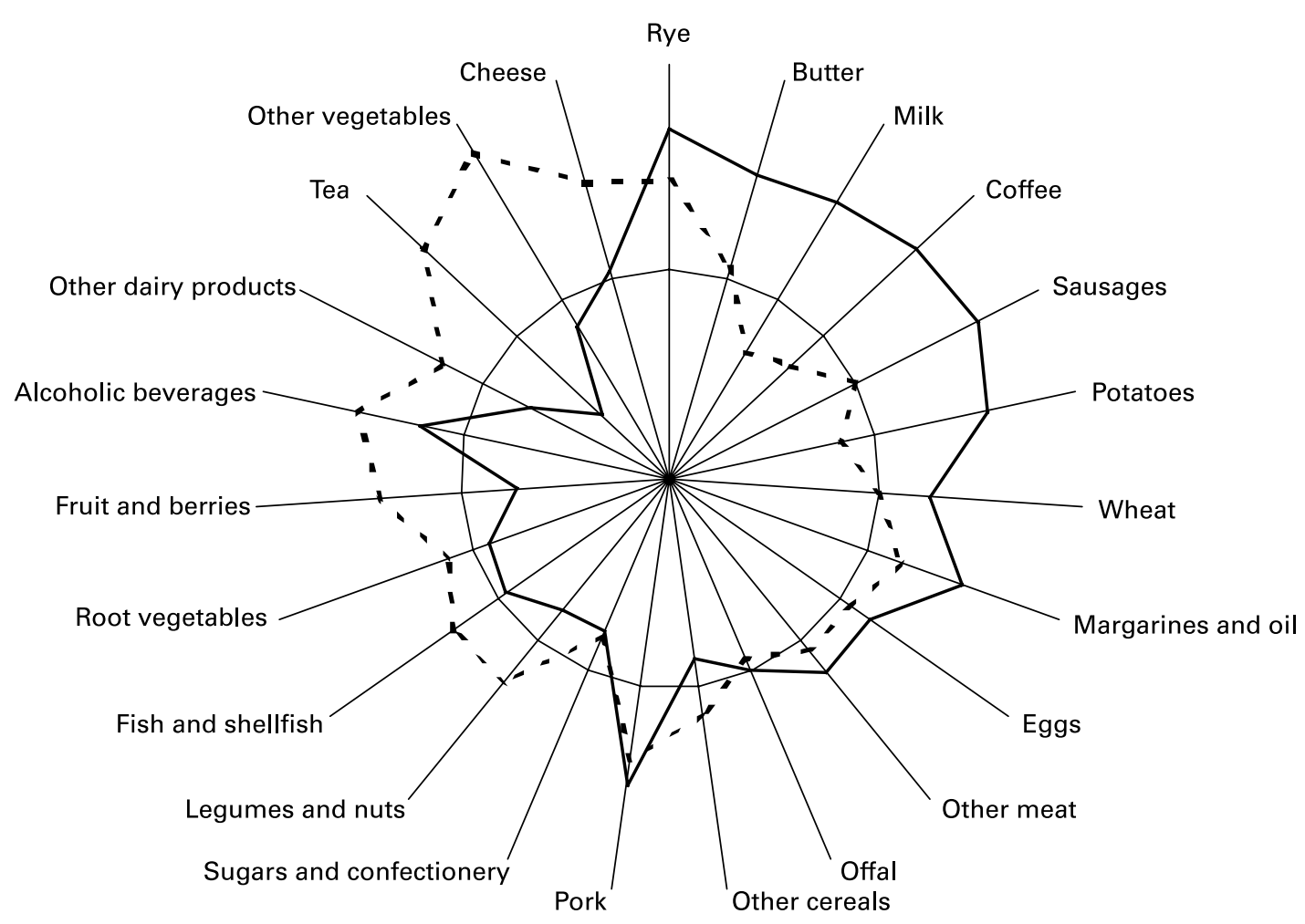

Fig. 1. Dietary patterns identified among subjects in 2001. Each arm of the star illustrates the correlation between the pattern and the food group, with an inverse correlation $(r-1)$ at the midpoint and a positive correlation $(r+1)$ at the outer edge of the constellation. A correlation of 0 is indicated by the circle. Very similar patterns were identified in 1980 and 1986. For details, see Mikkilä et al. ${ }^{(11)}$.

\section{Subjects and methods}

The Young Finns Study is a large, longitudinal multicentre study on CVD risk factors and their determinants among children, adolescents and young adults in Finland. Determinations have included ascertaining serum cholesterol and other lipid concentrations, blood pressure examinations, anthropometric measurements, and dietary interviews. Data have been collected with questionnaires on diet, smoking, alcohol use, physical activity, and other lifestyle factors, and on subjects' own and their family's socio-demographic situation and history.

The present study was conducted according to the guidelines laid down in the Declaration of Helsinki and all procedures involving human subjects were approved by the local ethics committees of the five study centres. Written informed consent was obtained from all subjects and/or from their guardians.

Details of the methods have been described previously ${ }^{(9,10)}$.

\section{Subjects}

The first cross-sectional study was carried out in 1980, when the participants were aged $3,6,9,12,15$ and 18 years ( $n$ 3596; $83 \%$ of those invited, randomly chosen from the national register) and the same subjects have been followed since. All of those participating in 1980 were re-invited in 1986 and in 2001 to the follow-up studies, and approximately 70 and $66 \%$ participated, respectively.

In 1980, a $50 \%$ random sample of the subjects was chosen to participate in the $48 \mathrm{~h}$ dietary recall interview.
These subjects were then repeatedly interviewed with the same method in subsequent follow-up studies. Dietary information and blood samples were obtained from 1768 subjects (then children and adolescents, aged 3-18 years) in 1980, from 1200 subjects (then children, adolescents and young adults, aged 9-24 years) in 1986, and from 1049 subjects (now all young adults, aged 24-39 years) in 2001. Complete dietary data from all study years and outcome data from 2001 were obtained from 785 subjects (420 women and 365 men). The characteristics of the subjects included in the present study are presented in Table 1.

\section{Dietary assessment and dietary patterns}

In the $48 \mathrm{~h}$ recall, dietary interviewers, all trained dietitians, collected information on foods and beverages consumed by subjects during the $2 \mathrm{~d}$ before the interview. In 1980 and 1986, 3- to 12-year-old children were interviewed together with their mother or father or another accompanying person. As detailed information as possible on the type and amount of foods and drinks reported was documented on forms by the interviewer. The study protocols have been described in detail elsewhere ${ }^{(13,14)}$. Details of the pattern analysis have also been reported previously ${ }^{(11)}$. Briefly, food groupings of the food consumption databases used in different years were unified to obtain twenty-three standardised food groups (Fig. 1). Food items were grouped according to their habitual culinary use or nutrient composition. Some items (for example, coffee, tea) were kept as a separate group because they were considered to represent distinctive food choices. 
Table 1. Characteristics of subjects in the year 2001

(Mean values and standard deviations or percentages)

\begin{tabular}{|c|c|c|c|c|}
\hline & \multicolumn{2}{|c|}{$\begin{array}{l}\text { Women } \\
(n \text { 420) }\end{array}$} & \multicolumn{2}{|c|}{ Men ( $n$ 365) } \\
\hline & Mean & SD & Mean & SD \\
\hline \multicolumn{5}{|l|}{ Age (years) } \\
\hline Mean & $30 \cdot 4$ & & $30 \cdot 1$ & \\
\hline Range & $24-39$ & & $24-39$ & \\
\hline \multicolumn{5}{|l|}{ Education (\%) } \\
\hline 9 years or less & \multicolumn{2}{|c|}{5} & \multicolumn{2}{|c|}{7} \\
\hline $10-12$ years & \multicolumn{2}{|c|}{21} & \multicolumn{2}{|c|}{34} \\
\hline 13 or more years & \multicolumn{2}{|c|}{74} & \multicolumn{2}{|c|}{59} \\
\hline \multicolumn{5}{|l|}{ Physical activity (\%) } \\
\hline Four or more times per week & \multicolumn{2}{|c|}{13} & \multicolumn{2}{|c|}{29} \\
\hline One to three times per week & \multicolumn{2}{|c|}{64} & \multicolumn{2}{|c|}{59} \\
\hline Less than once per week & \multicolumn{2}{|c|}{23} & \multicolumn{2}{|c|}{12} \\
\hline \multicolumn{5}{|l|}{ Smoking (\%) } \\
\hline Never & \multicolumn{2}{|c|}{52} & \multicolumn{2}{|c|}{38} \\
\hline Formerly or infrequently & \multicolumn{2}{|c|}{23} & \multicolumn{2}{|c|}{25} \\
\hline Currently & \multicolumn{2}{|c|}{25} & \multicolumn{2}{|c|}{37} \\
\hline $\begin{array}{c}\text { Maximum intima media } \\
\text { thickness }(\mathrm{mm})\end{array}$ & $0 \cdot 61$ & $0 \cdot 10$ & 0.63 & $0 \cdot 12$ \\
\hline Energy intake (MJ/d) & $7 \cdot 7$ & $2 \cdot 4$ & $10 \cdot 4$ & $3 \cdot 1$ \\
\hline Systolic blood pressure (mmHg) & 111 & 13 & 121 & 14 \\
\hline Total cholesterol (mmol/l) & 5.09 & 0.93 & $5 \cdot 21$ & 0.95 \\
\hline LDL-cholesterol (mmol/l) & $3 \cdot 15$ & 0.79 & 3.40 & 0.92 \\
\hline HDL-cholesterol (mmol/l) & $1 \cdot 40$ & 0.31 & $1 \cdot 16$ & 0.28 \\
\hline TAG $(\mathrm{mmol} / \mathrm{l})$ & $1 \cdot 20$ & 0.76 & 1.48 & 0.88 \\
\hline BMI $\left(\mathrm{kg} / \mathrm{m}^{2}\right)$ & $24 \cdot 5$ & $4 \cdot 7$ & $25 \cdot 8$ & $4 \cdot 3$ \\
\hline
\end{tabular}

A principal component analysis was performed separately for each study year to assess the major dietary patterns existing in the study group. The factors were rotated by an orthogonal transformation and the number of factors was considered on the basis of the Eigen values and a scree plot analysis. For each study year, we eventually identified two consistent dietary patterns. The patterns were extracted separately for men and women, but as the obtained factors were very similar (data not shown), the final pattern analyses were conducted with both sexes combined. The validity of the dietary patterns has been evaluated and reported earlier ${ }^{(11)}$.

\section{Intima media thickness}

Carotid ultrasound studies were performed using Sequoia 512 ultrasound mainframes (Acuson, Mountain View, CA, USA) with a $13.0 \mathrm{MHz}$ linear array transducer, as previously described $^{(9)}$. The studies were carried out in five study centres with similar equipment simultaneously between September 2001 and January 2002. Ultrasound technicians scanned the left common carotid artery following a standardised protocol. The image was focused on the posterior (far) wall, and gain settings served to optimise the image quality. A resolution box function (zoom) was used to record an image $25 \mathrm{~mm}$ wide and $15 \mathrm{~mm}$ high. A magnified image was recorded from an angle that showed the greatest distance between the lumen-intima interface and the media-adventitia interface. A moving scan with a duration of $5 \mathrm{~s}$, which included the beginning of the carotid bifurcation and the common carotid artery, was recorded and stored in digital format on optical disks for subsequent off-line analysis. A single reader blinded to participants' details manually analysed digitally stored scans with ultrasonic callipers. From the $5 \mathrm{~s}$ clip image, the best-quality end-diastolic frame was selected (incident with the $\mathrm{R}$ wave on a continuously recorded electrocardiogram). From this image, at least four measurements of the common carotid far wall were taken approximately $10 \mathrm{~mm}$ proximal to the bifurcation to derive maximal carotid IMT. To assess the reproducibility of IMT measurements, sixty participants (a $2.5 \%$ random sample of the total study population) were re-examined 3 months after the initial visit. The betweenvisit $\mathrm{CV}$ of the IMT measurements was $6.4 \%$. To assess the reproducibility of the IMT image analysis, a second observer reanalysed 113 scans; the between-observer CV was $5.2 \%$.

\section{Assessment of other variables}

Factors considered possible confounding factors in the relationship between diet and IMT, i.e. physical activity, smoking, blood pressure, the concentration of serum LDLcholesterol and BMI, were assessed in 2001. The variable representing physical activity was categorised using information on the frequency of rigorous exercise of at least 30 min duration into three categories: (1) four times or more per week; (2) one, two or three times per week; and (3) less than once per week. Smoking status was classified as never, formerly/infrequently, or currently. Based on reported years of schooling, we established a variable with three categories representing the Finnish educational system: (1) up to 9 years; (2) from 10 to 12 years; and (3) more than 12 years of education. Blood pressure was measured with a randomzero sphygmomanometer by a nurse. The average of three measurements of systolic blood pressure was used. Serum LDL-cholesterol concentrations were determined enzymically from venous samples drawn after a $12 \mathrm{~h}$ fast. Details of the lipid assessments have been published earlier ${ }^{(14)}$. BMI was calculated as the participant's weight $(\mathrm{kg})$ divided by height $\left(\mathrm{m}^{2}\right)$, measured by the nurse.

\section{Statistical analyses}

A dietary pattern score was computed for each subject, for both dietary patterns and for all study years. The higher the pattern score for a subject, the more his or her diet resembles that particular dietary pattern. Scores were standardised for a mean of 0 and a standard deviation of 1, and energy-adjusted using the residual method ${ }^{(15)}$. The adjusted scores were then averaged for the three study years. After testing for linearity, we examined the relationship between average dietary pattern scores and IMT in linear regression models. With the adulthood carotid artery IMT (maximum value in $\mathrm{mm}$ ) as the outcome variable, we constructed three models for both dietary patterns. Model 1 included the standardised, energy-adjusted and averaged pattern score as the explanatory variable, controlling for age. In multivariable model 2, potential confounding factors, i.e. smoking, physical activity and education, were added. Systolic blood pressure, LDL concentrations and BMI were considered possible confounding factors, but also factors possibly in the causal pathway between diet and IMT, and therefore included only in model 3. All linear regression analyses on dietary pattern scores were carried out for women and men separately. 
The analyses were repeated after the exclusion of those taking antihypertensive or lipid-lowering medications in 2001 ( $n$ 19), with similar results. All analyses were carried out using version 9.1 for Windows of SAS statistical software (2005; SAS Institute Inc., Cary, NC, USA).

\section{Results}

The traditional pattern, identified earlier through factor analysis, was directly correlated with intakes of rye, potatoes, milk, butter, sausages and coffee in each study year. The other pattern, labelled as the health-conscious dietary pattern, was characterised by vegetables, legumes and nuts, rye, tea, cheese and other dairy products at all study points, and also by alcoholic beverages in 2001. Dietary patterns identified in 2001 are presented in Fig. 1; very similar patterns were observed also in 1980 and 1986. The details of the patterns have been described previously ${ }^{(11)}$. Briefly, high traditional pattern scores were more common among men, subjects living in rural areas, smokers and those with a lower level of education. High scores also indicated in both childhood and adulthood lower intakes of carbohydrates, sucrose and vitamin $\mathrm{C}$, and higher intakes of total and saturated fat. Subjects with a high score in the health-conscious pattern tended to be non-smokers, the well educated and urbanites. High health-conscious pattern scores indicated lower intakes of saturated fat and sucrose and higher intakes of polyunsaturated and $n-3$ fatty acids, fibre, alcohol and vitamin C.

We divided the participants into sex-specific tertiles according to the energy-adjusted pattern score averaged over all study years. The mean IMT values according to the crosstabulated pattern tertiles are represented in Fig. 2. The positive association between the traditional pattern score and IMT was more apparent among subjects with a simultaneous low health-conscious pattern score.

In the multivariate linear regression model, the energyadjusted traditional pattern score averaged over all study years was positively associated with IMT in both women (b 0.020 (SEE 0.008); $P<0.01$ ) and men (b 0.032 (SEE 0.007);

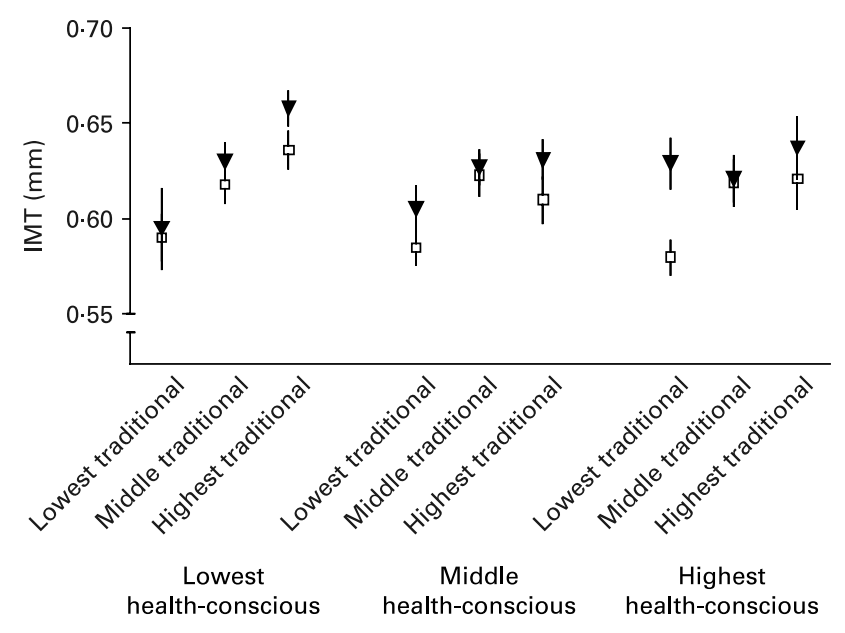

Fig. 2. Maximum intima media thickness (IMT) according to the combinations of the tertiles of the dietary pattern scores. Values are means for the lowest, middle and highest tertiles of the traditional and health-conscious pattern scores averaged for all study years for women $(\square)$ and men $(\boldsymbol{\nabla})$, with standard errors represented by vertical bars.
Table 2. Multivariable analyses of the longitudinal associations of dietary patterns with maximum carotid intima media thickness (women $n$ 420; men $n$ 365)

(Regression coefficients and standard error estimates)

\begin{tabular}{|c|c|c|c|c|c|c|}
\hline & \multicolumn{3}{|c|}{ Traditional dietary pattern* } & \multicolumn{3}{|c|}{$\begin{array}{l}\text { Health-conscious dietary } \\
\text { pattern* }\end{array}$} \\
\hline & $\mathrm{b} \dagger$ & SEE & $P$ & $\mathrm{~b} \dagger$ & SEE & $P$ \\
\hline \multicolumn{7}{|l|}{ Model 1‡ } \\
\hline Women & 0.020 & 0.008 & $<0.01$ & 0.017 & 0.006 & 0.06 \\
\hline Men & 0.032 & 0.007 & $<0.01$ & 0.004 & 0.006 & 0.49 \\
\hline \multicolumn{7}{|l|}{ Model $2 \S$} \\
\hline Women & 0.006 & 0.009 & 0.52 & 0.005 & 0.006 & 0.25 \\
\hline Men & 0.022 & 0.007 & $<0.01$ & -0.003 & 0.006 & 0.54 \\
\hline \multicolumn{7}{|l|}{ Model 3\| } \\
\hline Women & 0.003 & 0.007 & 0.66 & 0.006 & 0.006 & 0.26 \\
\hline Men & 0.019 & 0.006 & $<0.01$ & -0.002 & 0.005 & 0.76 \\
\hline
\end{tabular}

* Pattern score averaged over all study years. Traditional pattern score ranges from -1.9 to $5 \cdot 3$; health-conscious pattern score ranges from -3.8 to 6.9 .

tRegression coefficient $b$ indicates the increase in the predicted value of the outcome variable $(\mathrm{mm})$ for $1 \mathrm{SD}$ increase in the pattern score, keeping other variables in the model fixed.

$\ddagger$ Model 1: adjusted for age (years) and total energy (kJ).

$\S$ Model 2: additional adjustment for smoking (never, formerly or infrequently, currently), physical activity (four or more times per week; one, two or three times per week; less than once per week) and education (9 years or less; 10-12 years; 13 years or more).

I| Model 3: additional adjustment for systolic blood pressure $(\mathrm{mmHg})$, LDL-cholesterol $(\mathrm{mmol} / \mathrm{l})$ and $\mathrm{BMl}\left(\mathrm{kg} / \mathrm{m}^{2}\right)$.

$P<0 \cdot 01$ ) (Table 2). After adjusting with possible confounding factors, the association disappeared in women, but remained highly significant in men $(P<0 \cdot 01)$. Health-conscious dietary pattern scores showed no significant associations with IMT. On the basis of 2001 data, the correlations between the consumption of separate food groups and IMT were also calculated (data not shown). Of the twenty-three food groups, only the consumptions of rye, meat and coffee were significantly $(P<0 \cdot 05)$ associated with IMT, all positively.

\section{Discussion}

The main goal of the present study was to investigate the role of the diet as a combination of beneficial, adverse and neutral effects on IMT. Based on two previously identified, longitudinally fairly consistent dietary patterns, we found the traditional dietary pattern to be significantly associated with increased carotid IMT in men. This is in concordance with our earlier results showing direct associations between the traditional dietary pattern score and several cardiovascular risk factors $^{(12)}$. Foods characterising traditional diet have numerous compounds that may contribute to the health effects of the whole diet. While some of the effects may act via established risk factors, such as serum lipids, it is also possible that the diet also has other, independent influence.

Dietary pattern analyses have become increasingly popular in nutritional epidemiology. The dietary pattern approach, especially when carried out using exploratory factor analysis, is strongly dependent on cultural and behavioural factors within the study population, and therefore not generalisable to other populations. However, the identified patterns and many of the findings in studies on dietary patterns and CVD have revealed surprisingly similar results. In most cases, patterns including high amounts of vegetables and fruits, 
often labelled as 'healthy' or 'prudent', have been shown to be protective against $\mathrm{CVD}^{(4,5,16)}$, whereas patterns characterised by red and processed meat, refined grains and high-fat foods, labelled as a 'Western', 'empty calorie' or 'animal food' pattern, seem to be directly associated with CVD or its risk factors ${ }^{(6,7)}$. This Western type of diet consists of deep-fried foods, hamburgers, pizza and soft drinks, i.e. foods very different from the Finnish traditional pattern, but the two diets do have similar nutrient contents. They are both characterised by an elevated intake of high-fat and high-Na foods that are often low in antioxidative nutrients such as vitamin $\mathrm{C}$, folate and carotenes. Although our health-conscious dietary pattern resembles the 'healthy' or 'prudent' pattern observed in other studies, it did not show independent effects on carotid IMT. One possible explanation for this is that subjects with high scores in the healthconscious pattern had marked variation in the nutrient contents of their diets. This may be due to the long time frame of the present study; food selection and nutrient content of many common foods have changed during the 21 years' follow-up, and taking all changes into account in our analyses was not possible. However, the present results showed stronger associations between traditional diet and IMT among subjects with a low long-term health-conscious pattern score. This result suggests that these diets were inevitably low in many heart-healthy components that could have compensated for the harmful effects of the traditional diet.

The significant association between the longitudinal traditional dietary pattern score and carotid IMT observed in women disappeared after controlling for possible confounding factors. In this cohort, many of the risk factors found to be significant determinants of early vascular changes in men have not been shown to be equally manifested among women after adjusting for confounding factors ${ }^{(9)}$. This may be mostly due to the protective effect of endogenous oestrogen against atherosclerosis in premenopausal women ${ }^{(17,18)}$, but the results from intervention studies are inconsistent and the underlying mechanisms are not entirely clear ${ }^{(19)}$.

Although many of the observed associations can at least partly be attributed to the known effects of nutrients in the pattern diets, it is extremely difficult, if not impossible, to discuss the contribution of single nutrients or of single food items to the associations found. In a separate inspection, only three of the twenty-three food groups were significantly associated with early vascular changes as individual variables. These three (rye, meat and coffee) were also strongly loaded on the traditional dietary pattern. Therefore, it is unlikely that these foods would account for the association, but are to be considered indicators of wide-ranging traditional food choices and a traditional diet as a whole. Rye, in particular, has been linked to a lowered risk for CVD in studies carried out in Finland ${ }^{(20,21)}$. In Finland and many other countries in the Northern hemisphere, rye contributes a great part of the intakes of fibre, folate and phyto-oestrogens and is likely to have beneficial rather than adverse health effects. Indeed, rather than drawing conclusions of the effects of individual foods and food groups, the whole-diet approach is more efficient in showing the overall view of the relationship between food choices and the development of atherosclerosis. Moreover, the association found between the traditional dietary pattern and the IMT is stronger than that of the individual food groups, suggesting not only additive but also synergistic effects. One must bear in mind, however, that despite their advantages, dietary patterns are unlikely to predict a multifactorial group of diseases, such as CVD, solely by themselves. Even if a pattern has been shown to be associated with CVD, one cannot conclude that the observed pattern is the most beneficial or most detrimental of all possible diets ${ }^{(22)}$. However, recognising existing dietary patterns and their relationships with health outcomes in a given population may be useful in understanding the diet-disease phenomenon within the relevant, population-specific context and in identifying susceptible dietary behaviour.

The present study has several limitations that need to be taken into account. In a prospective setting with a long follow-up period, any selection bias occurs already at baseline and a possible loss during follow-up should be considered. The participation rate in 1980 was $83 \%$, considered sufficiently high to draw generalisable conclusions ${ }^{(10)}$. A later analysis revealed no clear differences in cardiovascular risk factors between subjects who had been lost to follow-up after 1980 and those who participated also in 2001, i.e. $66 \%$ of those invited ${ }^{(10)}$. In conclusion, any selection bias that might have occurred is not likely to undermine the group differences and associations found.

The present results may have been affected by residual confounding that we have not been able to rule out. In addition, most of the confounding factors that have been controlled for have been measured cross-sectionally, i.e. at the same time with the outcome. When examining the effects of whole diets on health outcomes, it is difficult to offer comprehensive biological explanations because of the numerous dietary factors that can act individually, in combinations, and/or in interactions with each other. We believe that dietary pattern analyses and other whole-diet approaches are most useful in identifying those diets that pose a risk to cardiovascular health over the long run.

Our analyses are based on food consumption information obtained by the $48 \mathrm{~h}$ recall method, which fails to take into account intra-individual variability in the diet ${ }^{(15)}$. However, we used information from three study years, thus being able to partly overcome this limitation. Moreover, the different dietary patterns were mostly distinguished by foods typically consumed daily or several times per week, such as coffee, tea, potatoes, butter, bread, vegetables and fruit. Earlier, we used the results of a FFQ filled out by the same participants to evaluate the validity of the dietary patterns ${ }^{(11)}$ and showed high correlations between food consumption results obtained with these two methods. Similar findings of dietary patterns derived using different methods have been presented for other populations ${ }^{(23,24)}$. Our previous study also showed substantial tracking of food choices assessed with pattern analysis from childhood to adulthood ${ }^{(11)}$.

The Cardiovascular Risk in Young Finns Study is one of the largest follow-up studies of cardiovascular risk from childhood to adulthood. Its strengths include a long followup time, a population-based setting from childhood onwards, a high participation rate, and a comprehensive, multidisciplinary approach. Aiming to achieve a broad picture of the development of CVD, the present study provides new knowledge on the relationship between diet and early atherosclerosis, assessed by carotid IMT. 
In conclusion, long-term adherence to traditional food choices seems to increase the risk of developing CVD among Finnish men.

\section{Acknowledgements}

The present study was financially supported by the Academy of Finland (grants no. 77841, 210283 and 117832), Turku University Hospital (federal grants), Juho Vainio Foundation, Finnish Foundation of Cardiovascular Research, Yrjö Jahnsson Foundation and Turku University Foundation.

V. M., L. R. and M. M. L. L. designed the present study. V. M. was responsible for the statistical analyses and drafted the manuscript. O. T. R. and J. V. are the responsible senior investigators in the Cardiovascular Risk in Young Finns Study. M. J. analysed the IMT scans. L. R. and V. M. were responsible for the dietary assessments. All authors participated in editing the final manuscript and approved the final version.

None of the authors has conflicts of interest.

\section{References}

1. Raitakari OT, Juonala M, Rönnemaa T, et al. (2008) Cohort profile: The Cardiovascular Risk in Young Finns Study. Int J Epidemiol 37, 1220-1226.

2. Moeller SM, Reedy J, Millen AE, et al. (2007) Dietary patterns: challenges and opportunities in dietary patterns research an experimental biology workshop, April 1, 2006. J Am Diet Assoc 107, 1233-1239.

3. Serra-Majem L, Roman B \& Estruch R (2006) Scientific evidence of interventions using the Mediterranean diet: a systematic review. Nutr Rev 64, 27-47.

4. Huijbregts PP, Feskens EJ \& Kromhout D (1995) Dietary patterns and cardiovascular risk factors in elderly men: the Zutphen Elderly Study. Int J Epidemiol 24, 313-320.

5. Millen BE, Quatromoni PA, Nam BH, et al. (2002) Dietary patterns and the odds of carotid atherosclerosis in women: the Framingham Nutrition Studies. Prev Med 35, 540-547.

6. van Dam RM, Rimm EB, Willett WC, et al. (2002) Dietary patterns and risk for type 2 diabetes mellitus in U.S. men. Ann Intern Med 136, 201-209.

7. Nettleton JA, Steffen LM, Schulze MB, et al. (2007) Associations between markers of subclinical atherosclerosis and dietary patterns derived by principal components analysis and reduced rank regression in the Multi-Ethnic Study of Atherosclerosis (MESA). Am J Clin Nutr 85, 1615-1625.

8. Simon A, Gariepy J, Chironi G, et al. (2002) Intima-media thickness: a new tool for diagnosis and treatment of cardiovascular risk. J Hypertens 20, 159-169.

9. Raitakari OT, Juonala M, Kähönen M, et al. (2003) Cardiovascular risk factors in childhood and carotid artery intima-media thickness in adulthood: the Cardiovascular Risk in Young Finns Study. JAMA 290, 2277-2283.

10. Juonala M, Viikari JS, Hutri-Kähönen N, et al. (2004) The 21-year follow-up of the Cardiovascular Risk in Young Finns Study: risk factor levels, secular trends and east-west difference. J Intern Med 255, 457-468.

11. Mikkilä V, Räsänen L, Raitakari OT, et al. (2005) Consistent dietary patterns identified from childhood to adulthood: The Cardiovascular Risk in Young Finns Study. Br J Nutr 93, 923-931.

12. Mikkilä V, Räsänen L, Raitakari OT, et al. (2007) Major dietary patterns and cardiovascular risk factors from childhood to adulthood. The Cardiovascular Risk in Young Finns Study. Br J Nutr 98, 218-225.

13. Räsänen L, Ahola M, Kara R, et al. (1985) Atherosclerosis precursors in Finnish children and adolescents. VIII. Food consumption and nutrient intakes. Acta Paediatr Scand 318, S135-S153.

14. Mikkilä V, Räsänen L, Raitakari OT, et al. (2004) Longitudinal changes in the diet from childhood to adulthood with respect to cardiovascular diseases - The Cardiovascular Risk in Young Finns. Eur J Clin Nutr 58, 1038-1045.

15. Willett WC (1998) Nutritional Epidemiology, 2nd ed. New York: Oxford University Press.

16. Fung TT, Willett WC, Stampfer MJ, et al. (2001) Dietary patterns and the risk of coronary heart disease in women. Arch Intern Med 161, 1857-1862.

17. Rosano GM, Sarrel PM, Poole-Wilson PA, et al. (1993) Beneficial effect of oestrogen on exercise-induced myocardial ischaemia in women with coronary artery disease. Lancet $\mathbf{3 4 2}$, $133-136$.

18. Clarkson TB (2007) Estrogen effects on arteries vary with stage of reproductive life and extent of subclinical atherosclerosis progression. Menopause 14, 373-384.

19. ESHRE Capri Workshop Group (2006) Hormones and cardiovascular health in women. Hum Reprod Update 12, 483-497.

20. Pietinen P, Rimm EB, Korhonen P, et al. (1996) Intake of dietary fiber and risk of coronary heart disease in a cohort of Finnish men. The Alpha-Tocopherol, Beta-Carotene Cancer Prevention Study. Circulation 94, 2720-2727.

21. Leinonen KS, Poutanen KS \& Mykkänen HM (2000) Rye bread decreases serum total and LDL cholesterol in men with moderately elevated serum cholesterol. J Nutr 130, $164-170$.

22. Schulze MB \& Hu FB (2002) Dietary patterns and risk of hypertension, type 2 diabetes mellitus, and coronary heart disease. Curr Atheroscler Rep 4, 462-467.

23. Togo P, Heitmann BL, Sorensen TI, et al. (2003) Consistency of food intake factors by different dietary assessment methods and population groups. Br J Nutr 90, 667-678.

24. McNaughton SA, Mishra GD, Bramwell G, et al. (2005) Comparability of dietary patterns assessed by multiple dietary assessment methods: results from the 1946 British Cohort. Eur J Clin Nutr 59, 341-352. 